\title{
The Diversity of Cosmic Explosions: $\gamma$-Ray Bursts and Type Ib/c Supernovae
}

\author{
Edo Berger \\ Division of Physics, Mathematics and Astronomy, California Institute of Technology, \\ Pasadena, CA 91125
}

\begin{abstract}
The death of massive stars and the processes which govern the formation of compact remnants are not fully understood. Observationally, this problem may be addressed by studying different classes of cosmic explosions and their energy sources. Here we discuss recent results on the energetics of $\gamma$-ray bursts (GRBs) and Type Ib/c Supernovae (SNe Ib/c). In particular, radio observations of GRB 030329, which allow us to undertake calorimetry of the explosion, reveal that some GRBs are dominated by mildly relativistic ejecta such that the total explosive yield of GRBs is nearly constant, while the ultra-relativistic output varies considerably. On the other hand, SNe Ib/c exhibit a wide diversity in the energy contained in fast ejecta, but none of those observed to date (with the exception of SN 1998bw) produced relativistic ejecta. We therefore place a firm limit of $3 \%$ on the fraction of SNe Ib/c that could have given rise to a GRB. Thus, there appears to be clear dichotomy between hydrodynamic (SNe) and engine-driven (GRBs) explosions.
\end{abstract}

\subsection{The Death of Massive Stars}

The death of massive stars $\left(M \gtrsim 8 \mathrm{M}_{\odot}\right)$ is a chapter of astronomy that is still being written. Recent advances in modeling suggests that a great diversity can be expected. Indeed, such diversity has been observed in the neutron star remnants: radio pulsars, AXPs, and SGRs. We know relatively little about the formation of black holes.

The compact objects form following the collapse of the progenitor core. The energy of the resulting explosion can be supplemented or even dominated by the energy released from the compact object (e.g. a rapidly rotating magnetar or an accreting black hole). Such "engines" can give rise to asymmetrical explosions (MacFadyen \& Woosley 1999), but even in their absence the core collapse process appears to be mildly asymmetric (e.g. Wang et al. 
2001). Regardless of the source of energy, a fraction, $E_{K}$, is coupled to the debris or ejecta (mass $M_{\mathrm{ej}}$ ) and it is these two gross parameters which determine the appearance and evolution of the resulting explosion. Equivalently one may consider $E_{K}$ and the mean initial speed of ejecta, $v_{0}$, or the Lorentz factor, $\Gamma_{0}=\left[1-\beta_{0}^{2}\right]^{-1 / 2}$, where $\beta_{0}=v_{0} / c$.

Supernovae (SNe) and $\gamma$-ray bursts (GRBs), are distinguished by their ejecta velocities. In the former $v_{0} \sim 10^{4} \mathrm{~km} \mathrm{~s}^{-1}$ as inferred from optical absorption features (e.g. Filippenko 1997), while for the latter $\Gamma_{0} \gtrsim 100$, inferred from the non-thermal prompt emission (Goodman 1986; Paczynski 1986), respectively. The large difference in initial velocity arises from significantly different ejecta masses: $M_{\mathrm{ej}} \sim$ few $\mathrm{M}_{\odot}$ in SNe compared to $\sim 10^{-5}$ $\mathrm{M}_{\odot}$ in GRBs.

In the conventional interpretation, $M_{\mathrm{ej}}$ for $\mathrm{SNe}$ is large because $E_{K}$ is primarily derived from the (essentially) symmetrical collapse of the core and the energy thus couples to all the mass left after the formation of the compact object. GRB models, on the other hand, appeal to a stellar mass black hole remnant, which accretes matter on many dynamical timescales and powers relativistic jets (the so-called collapsar model; MacFadyen \& Woosley 1999).

Still, as demonstrated by the association of the energetic Type Ic SN 1998bw $(d \approx 40 \mathrm{Mpc}$ ) with GRB 980425 (Galama et al. 1998), as well as the association of SN 2003dh with GRB 030329 (e.g. Stanek et al. 2003), some overlap may exist. Here we take an observational approach to investigating the diversity of stellar explosions, their energetics, and the relation between them focusing in particular on GRBs and $\mathrm{SNe} \mathrm{Ib} / \mathrm{c}$.

\subsection{The Energetics of $\gamma$-Ray Bursts}

Recent studies revealed the surprising result that long-duration GRBs have a standard energy of $E_{\gamma} \approx 1.3 \times 10^{51}$ erg in ultra-relativistic ejecta when corrected for asymmetry ("jets"; Frail et al. 2001; Bloom, Frail \& Kulkarni 2003). A similar result was found for the kinetic energies of GRB afterglow using the beaming-corrected X-ray luminosities as a proxy for the true kinetic energy (Figure 1.1 Berger, Kulkarni \& Frail 2003). However, these studies have also highlighted a small group of sub-energetic bursts, including the peculiar GRB 980425 associated (Galama et al. 1998) with SN 1998bw $\left(E_{\gamma} \approx 10^{48} \mathrm{erg}\right)$. Until recently, the nature of these sources has remained unclear.

This question appears to now be resolved thanks to broad-band calorimetry of GRB 030329 (Berger et al. 2003a), the nearest cosmological burst 
The Diversity of Cosmic Explosions: $\gamma$-Ray Bursts and Type Ib/c Supernovae 3
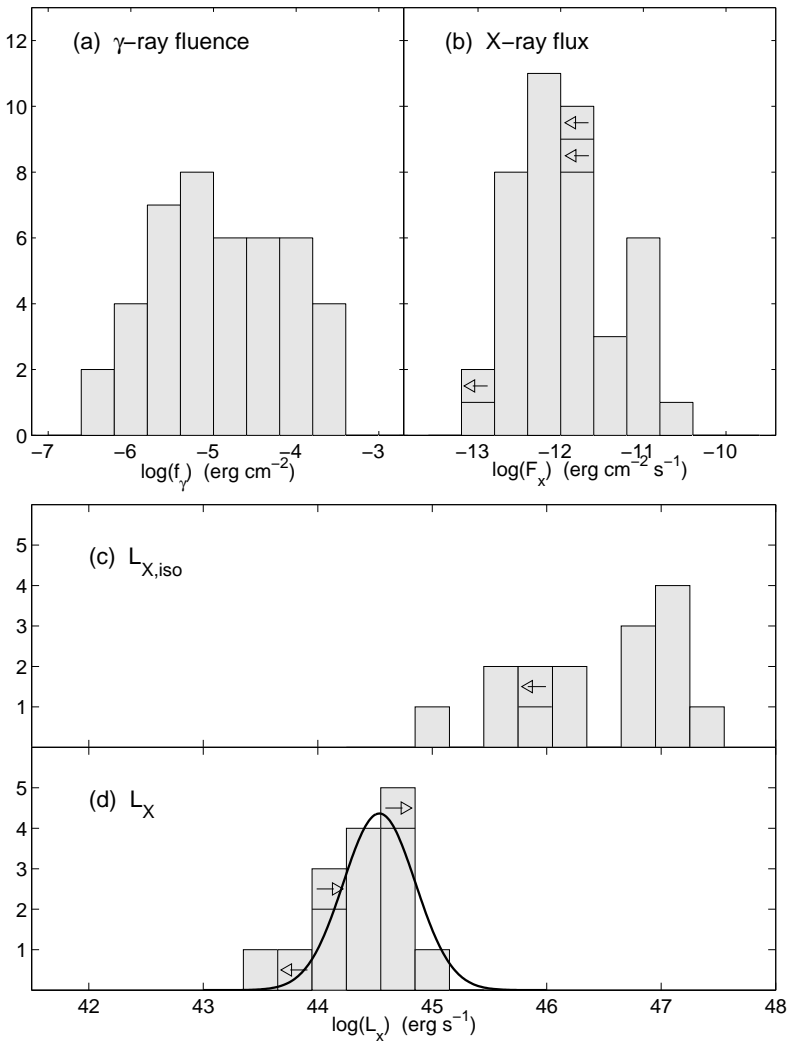

Fig. 1.1. (a) Distribution of $\gamma$-ray fluences; (b) Distribution of X-ray fluxes scaled to $t=10 \mathrm{hr}$ after the burst; (c) Isotropic-equivalent X-ray luminosity plotted for the subset of X-ray afterglows with known jet opening angles and redshifts; (d) True $\mathrm{X}$-ray luminosity corrected for beaming, a proxy for the afterglow kinetic energy.

detected to date (redshift, $z=0.1685$ ). Early optical observations of the afterglow of GRB 030329 revealed a sharp break at $t=0.55$ day (Figure 1.2 Price et al. 2003). The X-ray flux (Tiengo et al. 2003) tracks the optical afterglow for the first day, with a break consistent with that seen in the optical. Thus, the break at 0.55 day is not due to a change in the ambient density since for typical parameters (e.g. Kumar 2000) the X-ray emission is not sensitive to density. However, unlike the optical emission the X-ray flux at later time continues to decrease monotonically.

Given the characteristic $F_{\nu} \propto t^{-2}$ decay for both the X-ray and optical emission beyond 0.55 day, the break is reasonably modeled by a jet with an opening angle of $5^{\circ}$. The inferred beaming-corrected $\gamma$-ray energy is only $E_{\gamma} \approx 5 \times 10^{49} \mathrm{erg}$, significantly lower than the strong clustering around 


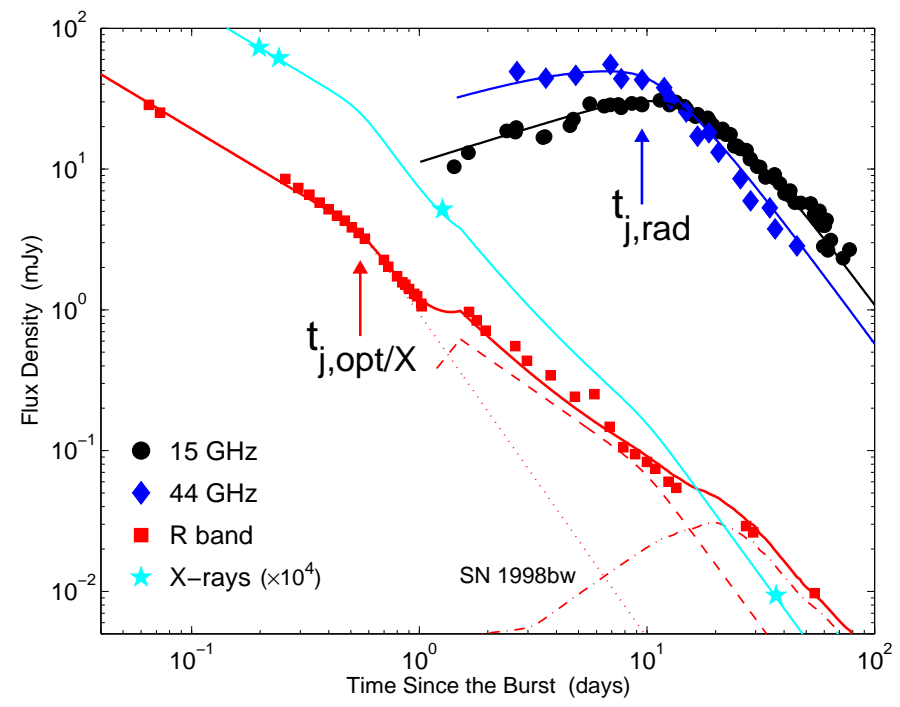

Fig. 1.2. Radio to X-ray lightcurves of the afterglow of GRB 030329, exhibiting the early jet break at 0.55 days in the optical and X-ray bands, as well as the subsequent rise in optical flux and the bright radio emission arising from a jet with an opening angle of $17^{\circ}$. Also plotted is the optical emission from SN 1998bw at the redshift of GRB 030329 as a proxy for SN 2003dh. The solid line is a combination of all the different emission components.

$1.3 \times 10^{51}$ erg seen in most bursts. Similarly, the beaming-corrected X-ray luminosity at $t=10$ hours is $L_{X, 10} \approx 3 \times 10^{43} \mathrm{erg} \mathrm{s}^{-1}$, a factor of ten below the tightly clustered values for "typical" bursts (Figure 1.11).

The radio afterglow of GRB 030329 (Berger et al. 2003a; Sheth et al. 2003) reveals a different picture. The increase in flux during the first 10 days, followed by a rapid decline, $F_{\nu} \propto t^{-1.9}$ at $t \gtrsim 10$ day and a decrease in peak flux at $\nu \lesssim 22.5 \mathrm{GHz}$ (Figure 1.3) are indicative of a jet with an opening angle of $17^{\circ}$. The inferred beaming-corrected kinetic energy in the explosion is about $3 \times 10^{50} \mathrm{erg}$, comparable to what is inferred from modeling of other afterglows (Panaitescu \& Kumar 2002).

This result, combined with the resurgence in the optical emission at 1.5 days, is best explained in the context of a two-component explosion model. In this scenario the first component (a narrow jet, $5^{\circ}$ ) with initially a larger Lorentz factor is responsible for the $\gamma$-ray burst and the early optical and X-ray afterglow including the break at 0.55 day, while the second component (a wider jet, $17^{\circ}$ ) powers the radio afterglow and late optical emission (Figure 1.2 Berger et al. 2003a). The break at 10 days due to the second component has recently been inferred in the optical bands following a care- 
The Diversity of Cosmic Explosions: $\gamma$-Ray Bursts and Type Ib/c Supernovae 5

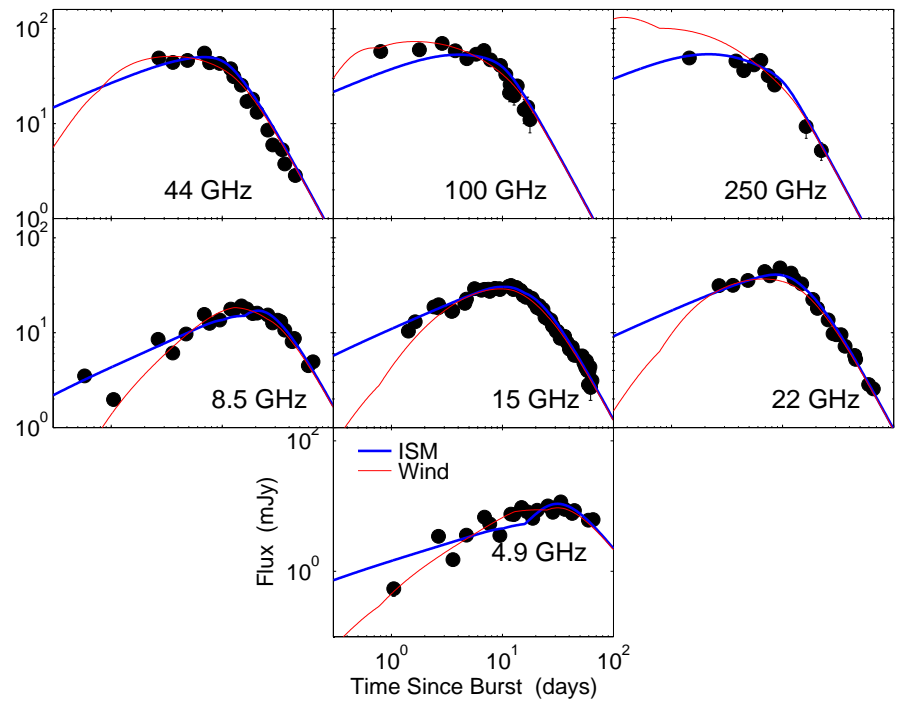

Fig. 1.3. Detailed radio lightcurves of the afterglow of GRB 030329. The solid lines are afterglow models of collimated ejecta expanding into circumburst media with a uniform and wind $\left(\rho \propto r^{-2}\right)$ density profiles. The sharp turnover at about 10 days is indicative of a jet with an opening angle of $17^{\circ}$.

ful subtraction of the light from SN 2003dh which accompanied GRB 030329 (e.g. Matheson et al. 2003). Such a two-component jet finds a natural explanation in the collapsar model (Zhang, Woosley \& Heger 2003).

The afterglow calorimetry of GRB 030329 has important ramifications for our understanding of GRB engines and the sub-energetic bursts. Namely, such bursts may have a total explosive yields similar to other GRBs (Figure 1.4), but their ultra-relativistic output varies considerably.

This leads to the following conclusions. First, radio calorimetry, which is sensitive to all ejecta with $\Gamma \gtrsim f e w$, shows that the total energy yield of GRB 030329 is similar to those estimated for other bursts. Along these lines, the enigmatic GRB 980425 associated with the nearby supernova SN 1998bw also has negligible $\gamma$-ray emission, $E_{\gamma \text {,iso }} \approx 8 \times 10^{47}$ erg; however, radio calorimetry (Li \& Chevalier 1999) showed that even this extreme event had a similar explosive energy yield (Figure 1.4). The newly recognized class of cosmic explosions, the X-ray Flashes, exhibit little or no $\gamma$-ray emission but appear to have comparable X-ray and radio afterglows to those of GRBs (see 1.4). Thus, the commonality of the total energy yield points to a common origin, but apparently the ultra-relativistic output is highly variable. Unraveling what physical parameter is responsible for this variation appears to be the next frontier in the field of cosmic explosions. 


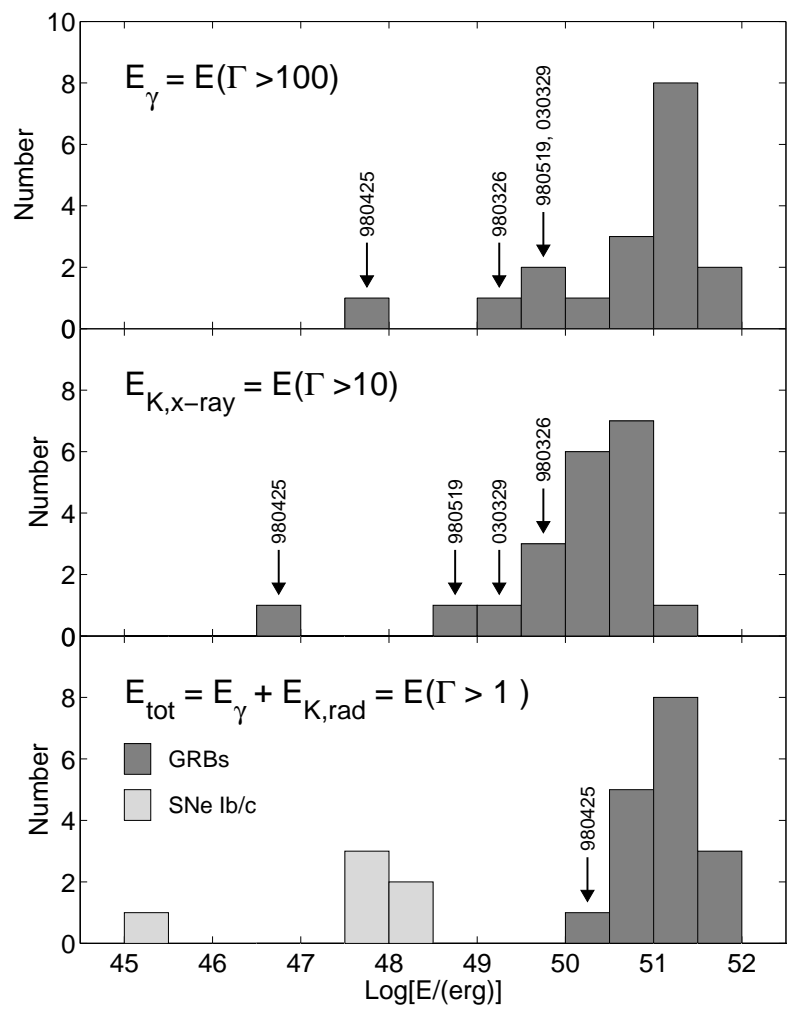

Fig. 1.4. The beaming-corrected energies of GRBs and $\mathrm{SNe} \mathrm{Ib} / \mathrm{c}$ in ejecta with a Lorentz factor ranging from $\gtrsim 100$ to order unity (top: the $\gamma$-ray energy, middle: the kinetic energy in the early afterglow; bottom: the total relativistic energy). The ultra-relativistic output of GRBs varies considerably despite a nearly standard total explosive yield. On the other hand, the significantly lower total energy in fast ejecta of $\mathrm{SNe} \mathrm{Ib} / \mathrm{c}$ points to a different energy source.

\subsection{The Incidence of Engine in Type Ib/c Supernovae}

The inferences summarized in the previous section, coupled with the association of some GRBs with SNe Ib/c raises the question: is there a population of SNe that is powered by engines? Observationally there appear to be many distinctions (e.g. ejecta velocity and mass), but the association of the Type Ic SN 1998bw with GRB 980425 has indicated that some overlap exists. In particular, the radio emission from SN 1998bw revealed mildly relativistic ejecta with a complex structure indicative of a long-lived energy source. The expected fraction of similar events in the local SN population, $\sim 0.5 \%$ to $25 \%$, depends on their origin: typical GRBs observed away from the jet axis versus an intermediate population of explosions. 
The Diversity of Cosmic Explosions: $\gamma$-Ray Bursts and Type Ib/c Supernovae 7

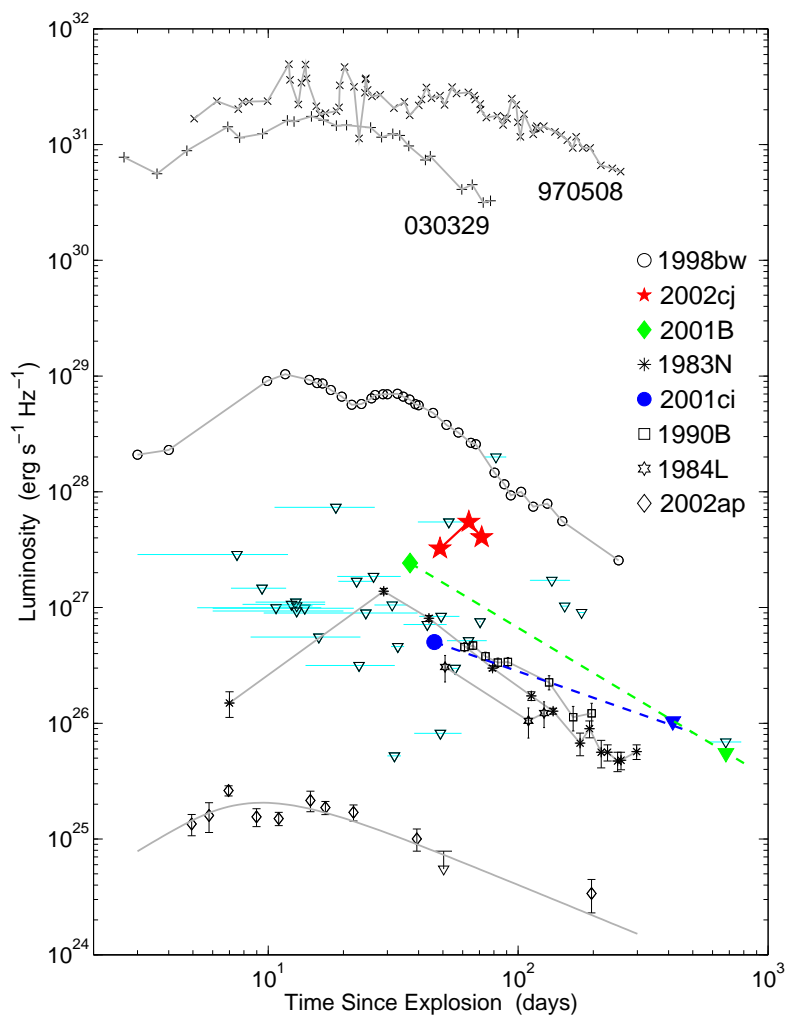

Fig. 1.5. Radio lightcurves of Type $\mathrm{Ib} / \mathrm{c} \mathrm{SNe}$ including the peculiar SN 1998bw/GRB 980425. A comparison to SN 1998bw and GRB afterglows reveals significantly less energy in high velocity ejecta, and thus constrains the fraction of $\mathrm{SNe} \mathrm{Ib} / \mathrm{c}$ that are powered by an engine to $<3 \%$. There is therefore a dichotomy in the explosion mechanism of massive stars.

To assess this fraction, and hence the origin of potential engine-driven SNe, directly, we have carried out since 1999 the most comprehensive radio survey of SNe Ib/c to date (Berger, Kulkarni \& Chevalier 2002; Berger et al. 2003b). As was demonstrated in the case of SN 1998bw, such observations provide the best probe of relativistic ejecta (a proxy for an engine).

As seen in Figure 1.5 the luminosity function of $\mathrm{SNe} \mathrm{Ib} / \mathrm{c}$ is significantly broader than previously inferred, but none of the observed SNe approach the luminosity of typical GRB afterglows. We therefore place a limit of about $3 \%$ on the fraction of local $\mathrm{SNe} \mathrm{Ib} / \mathrm{c}$ that are powered by an engine or potentially gave rise to a GRB (Berger et al. 2003b).

In the majority of cases we find expansion velocities of $\lesssim 0.3 c$ (Figure 1.6) as compared to $\Gamma \sim 2$ for SN 1998bw and $\Gamma \sim 5$ for GRB radio afterglows. 


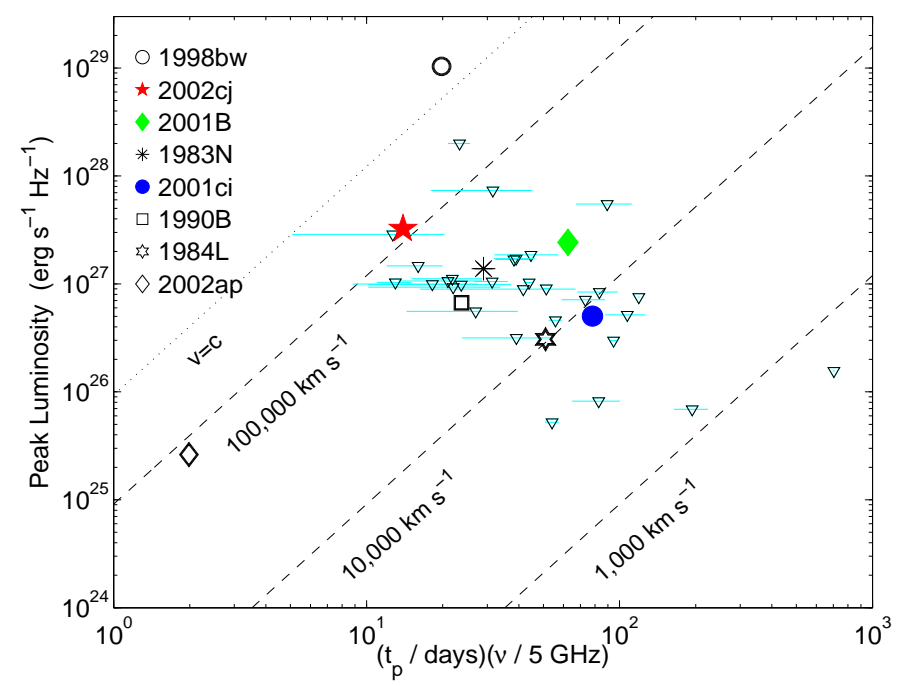

Fig. 1.6. Peak radio luminosity plotted versus the time of peak luminosity for Type $\mathrm{Ib} / \mathrm{c}$ SNe. The diagonal lines are contours of constant average expansion velocity based on the assumption that the peak of the radio luminosity occurs at the synchrotron self-absorption frequency.

Similarly, the energy carried by these ejecta can be accounted for in a hydrodynamic explosion model. In fact, as seen in Figure 1.4 SNe Ib/c are significantly less energetic in terms of fast ejecta compared to GRBs. Thus, GRBs and the vast majority of $\mathrm{SNe} \mathrm{Ib} / \mathrm{c}$ do not share a common energy source. We can therefore rule out models of GRBs or SNe which suggest a significant overlap (e.g. Lamb et al. 2003).

\subsection{Future Directions}

The recent recognition of a new class of cosmic explosion, the X-ray flashes (XRFs), provides an opportunity to extend the analysis presented above. These transients are defined as those with $\log \left[S_{X}(2-30 \mathrm{keV}) / S_{\gamma}(30-\right.$ $400 \mathrm{keV})]>0$, where $S_{X}$ and $S_{\gamma}$ are the fluences in the X-ray and $\gamma$-ray bands, respectively; i.e. the peak in the $\nu F_{\nu}$ spectrum lies in the X-ray band. With the exception of a significantly lower peak energy, XRFs share similar properties (e.g. duration, fluence) with GRBs.

Recent detections of XRF afterglows indicate that they likely arise at cosmological distances: they exhibit interstellar scintillation effects similar to those observed in GRB radio afterglows, they are associated with faint 
The Diversity of Cosmic Explosions: $\gamma$-Ray Bursts and Type Ib/c Supernovae 9

compact galaxies similar to the general population at $z \sim 1$, and in one case (XRF 020903), a redshift of 0.25 has been measured.

Using the measured redshift, the prompt energy release of XRF 020903 is only $\sim 2 \times 10^{49} \mathrm{erg}$, significantly lower than GRBs. The difference is even more pronounced when we consider that the spectrum peaked at $\sim 5 \mathrm{keV}$. Thus, it is possible that XRF 020903 (and perhaps all XRFs) produce a negligible amount of highly relativistic ejecta, maybe as a result of higher baryon contamination in the ejecta. However, preliminary results indicate that the total relativistic output of XRF 020903, measured from the radio afterglow in the usual manner, is similar to that of GRBs (Figure 1.4. Soderberg et al. 2003). If so, XRFs may in fact share a common origin with GRBs.

To assess this possibility it is crucial to obtain a large sample of XRFs with measured redshifts. This, along with continued monitoring of GRBs and their afterglows (especially in the Swift era) and continued radio observations of $\mathrm{SNe} \mathrm{Ib/c,} \mathrm{will} \mathrm{allow} \mathrm{us} \mathrm{to} \mathrm{determine} \mathrm{more} \mathrm{accurately} \mathrm{the} \mathrm{true} \mathrm{diversity}$ of cosmic explosions and the fraction of stellar deaths in each channel.

\section{Acknowledgments}

I would like to thank the conference organizers, C. Wheeler, P. Kumar, and P. Hoflich, and my many collaborators, in particular S. Kulkarni and D. Frail

\section{References}

Berger, E., Kulkarni, S. R., and Frail, D. A. 2003, ApJ, 590, 379.

Berger, E., et al. 2003a, Nature in press; astro-ph/0308187

Berger, E., Kulkarni, S. R., and Chevalier, R. A. 2002, ApJ, 577, L5.

Berger, E., et al. 2003b, ApJ in press; astro-ph/0307228

Bloom, J. S., Frail, D. A., and Kulkarni, S. R. 2003, astro-ph/0302210

Filippenko, A. V. 1997, ARA\&A, 35, 309.

Frail, D. A., et al. 2001, ApJ, 562, L55.

Galama, T. J., et al. 1998, Nature, 395, 670.

Goodman, J. 1986, ApJ, 308, L47.

Kumar, P. 2003, ApJ, 538, L125.

Li, Z. and Chevalier, R. A. 1999, ApJ, 526, 716.

MacFadyen, A. L. \& Woosley, S. E. 1999, ApJ, 524, 262.

Paczynski, B. 2001, Acta Astronomica, 51, 1.

Panaitescu, A. and Kumar, P. 2002, ApJ, 571, 779.

Price, P. A., et al. 2003, Nature, 423, 844.

Sheth, K., et al. 2003, ApJ, 595, L33.

Soderberg, A. M., et al. 2003, in prep.

Stanek, K. Z., et al. 2003, ApJ, 591, L17.

Tiengo, A., et al. 2003, astro-ph/0305564

Wang, L., et al. 2001, ApJ, 550, 1030.

Zhang, W., Woosley, S. E., \& Heger, A. 2003; astro-ph/0308389 